\title{
Whole brain radiotherapy with adjuvant or concomitant boost in brain metastasis: dosimetric comparison between helical and volumetric IMRT technique
}

Paolo Borghetti ${ }^{* *}$, Sara Pedretti ${ }^{1}$, Luigi Spiazzi ${ }^{2}$, Rossella Avitabile², Mauro Urpis ${ }^{1}$, Federica Foscarini ${ }^{1}$, Giulia Tesini ${ }^{2}$, Francesca Trevisan ${ }^{1}$, Paolo Ghirardelli ${ }^{1}$, Sara Angela Pandini ${ }^{1}$, Luca Triggiani ${ }^{1}$, Stefano Maria Magrini ${ }^{1}$

and Michela Buglione ${ }^{1}$

\begin{abstract}
Background: To compare and evaluate the possible advantages related to the use of VMAT and helical IMRT and two different modalities of boost delivering, adjuvant stereotactic boost (SRS) or simultaneous integrated boost (SIB), in the treatment of brain metastasis (BM) in RPA classes I-II patients.

Methods: Ten patients were treated with helical IMRT, 5 of them with SRS after whole brain radiotherapy (WBRT) and 5 with SIB. MRI co-registration with planning $C T$ was mandatory and prescribed doses were 30 Gy in 10 fractions (fr) for WBRT and 15Gy/1fr or 45Gy/10fr in SRS or SIB, respectively. For each patient, 4 "treatment plans" (VMAT SRS and SIB, helical IMRT SRS and SIB) were calculated and accepted if PTV boost was included in $95 \%$ isodose and dose constraints of the main organs at risk were respected without major deviations. Homogeneity Index ( $\mathrm{HI})$, Conformal Index $(\mathrm{Cl})$ and Conformal Number $(\mathrm{CN})$ were considered to compare the different plans. Moreover, time of treatment delivery was calculated and considered in the analysis.
\end{abstract}

Results: Volume of brain metastasis ranged between 1.43 and $51.01 \mathrm{cc}$ (mean $12.89 \pm 6.37 \mathrm{ml}$ ) and 3 patients had double lesions. V95\% resulted over $95 \%$ in the average for each kind of technique, but the "target coverage" was inadequate for VMAT planning with two sites. The HI resulted close to the ideal value of zero in all cases; VMAT-SIB, VMAT-SRS, Helical IMRT-SIB and Helical IMRT-SRS showed mean CI of 2.15, 2.10, 2.44 and 1.66, respectively (optimal range: 1.5-2.0). Helical IMRT-SRS was related to the best and reliable finding of CN (0.66). The mean of treatment time was 210 s, 467 s, 440 s, 1598 s, respectively, for VMAT-SIB, VMAT-SRS, Helical IMRT-SIB and Helical IMRT-SRS.

Conclusions: This dosimetric comparison show that helical IMRT obtain better target coverage and respect of $\mathrm{Cl}$ and CN; VMAT could be acceptable in solitary metastasis. SIB modality can be considered as a good choice for clinical and logistic compliance; literature's preliminary data are confirming also a radiobiological benefit for SIB. Helical IMRT-SRS seems less effective for the long time of treatment compared to other techniques.

Keywords: Brain metastases, Dosimetric comparison, Helical IMRT, Stereotactic radiotherapy, VMAT, Homogeneity index, Conformal index, Conformal number

\footnotetext{
* Correspondence: paolobor82@yahoo.it

${ }^{1}$ Radiation Oncology Department, University and Spedali Civili Brescia, P.le

Spedali Civili 1, Brescia, Italy

Full list of author information is available at the end of the article
} 


\section{Background}

Brain metastases occur in $30 \%$ of all patients with cancer [1] and the incidence is increasing in relation to the improvement of local control and survival related to new available systemic treatments and to the inability of most drugs to cross the blood brain barrier. Moreover, in Italy, the aging of the population and the better diagnostic accuracy obtained with Magnetic Resonance (MRI) contribute to the increase in frequency of BM diagnosis [2, 3].

The main prognostic factors for patients with BM are the Karnofski Performance Status (KPS) $(\geq 70$ or $<70$ ), age $(<65$ or $\geq 65)$, controlled primary site and the presence of extra cranial metastasis according to the Recursive Partitioning Analysis (RPA) [4, 5]. Recently, a Graded Prognostic Analysis (GPA) has been introduced in order to consider the histology of the primary tumour to the already known prognostic factors [6-8].

To date, the possible therapeutic approaches to BM are surgery (SUR) [9, 10], whole brain radiotherapy (WBRT) [11] and stereotactic radiosurgery (SRS) alone or in combination [7, 12]. Median overall survival (OS) is 1-2 month with supportive care [13], while after WBRT is 7.1, 4.2, 2.3 respectively for RPA class I, II, III [4]. More aggressive treatments as SUR or SRS alone or combined to WBRT can double OS rates [14] with an acceptable neurotoxicity and quality of life [15-17]. A careful patients selection remains crucial to identify who really benefits of intensified local treatments.

To date, it is widely debated if the linear quadratic (LQ) model is able to predict the biological effects of high fractional doses used for stereotactic treatment in BM. Kirkpatrick suggests that tumour control probability after a single stereotactic fraction is higher than expected according to the quadratic linear model [18]. On the contrary, Brenner argues that the LQ model is theoretically and experimentally validated up to a single fraction dose of 10 Gy [19]. Recently, it has been hypothesized that the introduction in the formalism of a parameter proportional to the cube of the dose (linear quadratic cubic or LQC- model), could result in a better fitting of the resulting dose - local control curve with the clinical data [20]. A revision of 11 studies on SRS in BM has shown a good correlation between the local control rate at 12 months and the biological equivalent dose ( $\alpha / \beta 12$ Gy), calculated with the LQC model, for fractional doses ranging between 6 and 25 Gy; the analysis concluded suggesting excellent local control rates at 12 months after single-fraction doses higher than 20 Gy as opposed to clearly insufficient results for doses lower than 15 Gy [21].

Some authors suggest that this approach does not allow to exploit all the potential benefits of fractionation in terms of radiobiological redistribution and re-oxygenation. Hall et al. argue that fractionated stereotactic treatments may be more effective than those in single fraction [22]. In addition, tumour cell repopulation and repair of sub-lethal damage may occur when a significant interval between WBRT and SRS is provided [23]. Finally, SRS treatments may require very long treatment sessions, possibly forcing to split them in multiple sessions.

From a dosimetric point of view, the possibility of incorporating the contribution of the boost in larger volumes treated with lower doses is a definite advantage of the simultaneous integrated boost (SIB) compared to SRS [24]. In fact, the stereotactic boost after WBRT adds an unintentional dose to the brain, while SIB technique makes possible to optimize the dose distribution taking into account WBRT and boost simultaneously $[25,26]$. Both volumetric modulated arc therapy (VMAT) and helical IMRT are able to deliver high radiation doses to the target with excellent precision, thus preserving organs at risk. Although it is not completely clear how SRS and SIB could be considered biologically equivalent due to the possible limits of applicability of LQ model, a comparative dosimetric and technical analysis of ten cases has been performed, in order to assess benefits or drawbacks of SRS vs SIB and Helical IMRT vs VMAT in terms of cost-effectiveness and appropriateness of different therapeutic techniques.

\section{Methods}

Ten patients with BM in RPA prognostic class I-II were considered for this analysis (in particular 5 with SRS and 5 with SIB). They were selected among the first patients treated with helical IMRT at our Department, in order to identify technical and dosimetric variables relevant in the treatment of BM with stereotactic approach and to compare helical IMRT with VMAT in this clinical setting. All patients had one BM but three of them who had two lesions. Five were treated with SRS after WBRT and the remaining 5 with SIB. SIB was preferred when MRI was available at planning, while SRS boost was postponed after WBRT in order to have MRI to confirm number and dimension of BM. All patients were treated with Helical IMRT. Precautionary dose of 15 Gy as SRS boost and 45 Gy as SIB were prescribed for these cases because they were the first patients treated in this way in our department.

Patients were simulated in supine position using a CT scan (3 mm slice thickness and spacing) over the entire head region; thermoplastic masks were used to immobilize the patients. CT images were co-registered through a rigid protocol with diagnostic MRI (axial T1-weighted gadolinium contrast enhancement sequences).

The whole brain was contoured and considered as WBCTV; different gross tumor volumes were defined for each metastasis as the contrast enhanced lesion; $5 \mathrm{~mm}$ radial and $6 \mathrm{~mm}$ cranio-caudal expansion was applied to 
obtain WB-PTV and $3 \mathrm{~mm}$ isotropic expansion to obtain boost-PTV.

Organs at risk (OAR) included eyes, lens, optic nerves, chiasm, brainstem and brain parenchyma (excluding BM).

A Helical IMRT dedicated treatment planning system (TPS) and the Pinnacle ${ }^{\bullet}$ V9.2 TPS were respectively used for Helical IMRT and VMAT plans.

VMAT plans were generated for an Elekta Synergy linear accelerator equipped with $1 \mathrm{~cm}$ at isocenter MLC. Two or more modulated arcs of $10 \mathrm{MV}$ nominal energy photons beams with maximum dose rate of 600 U.M./min were used (number of arcs used for SRS and SIB VMAT plans are reported in Table 2). Computing grid was of $2 \times 2 \times$ $3 \mathrm{~mm} 3$. Helical IMRT plans employed the standard nominal energy $6 \mathrm{MV}$ photons beams with either $1.05 \mathrm{~cm}$ field width or $2.5 \mathrm{~cm}$ field width for SRS boost and SIB respectively. Pitch value was defined 0.1 for SRS and 0.278 for concomitant boost plans. A modulation factor of 2.0 and a computing grid of $1.95 \times 1.95 \times 3 \mathrm{~mm} 3$ (fine calculation) were chosen for optimization process and final dose calculation for both the SRS boost and the SIB. All VMAT plans were coplanar with a single isocenter, even for two lesions ones.

All plans were elaborated by the same planner either for VMAT and Helical IMRT and all of them were re-planned by a second independent expert planner in order to ensure optimization.

For each of the 10 cases, 4 treatment plans were calculated and optimized (one of them was actually delivered).

Plan 1: WBRT (30 Gy/10 fr) followed by SRS boost (15 Gy/1 fr) with Helical IMRT technique

Plan 2: WBRT (30 Gy/10 fr) with SIB up to $45 \mathrm{~Gy} / 10 \mathrm{fr}$ with Helical IMRT technique

Plan 3: WBRT (30 Gy/10 fr) followed by SRS boost (15 Gy/1 fr) with VMAT technique

Plan 4: WBRT (30 Gy/10 fr) with SIB up to $45 \mathrm{~Gy} / 10 \mathrm{fr}$ with VMAT technique.

In the treatment plans with sequential boost, WBRT was provided using two $6 \mathrm{MV}$ opposing lateral fields equally balanced. In these cases Helical IMRT and VMAT were used exclusively for the boost. The dosimetric assessment of the organs at risk was performed considering the sum of the doses delivered in the two separate plans (WBRT + SRS boost). Regarding SIB plans, instead, the helical and volumetric techniques were used for the overall plan, to include whole brain and BM. All plans were elaborated in order to obtain the best potentially deliverable plan for an acceptable target coverage, without violations of dose constraints to OAR.

\section{Statistical analysis}

Doses to WB-PTV and boost-PTV were prescribed to $100 \%$ according to the criteria of the International Commission on Radiation Units \& Measurements (ICRU).
The minimum accepted dose to PTV brain and PTV boost was $95 \%$ of the prescribed dose. None maximum dose limit to PTV boost was established, although it was normally within the $108 \%$ of the prescribed dose.

The doses to the organs at risk were evaluated considering dose-constraints reported in the literature [25-27] (Table 1). Dose constraint to brain without PTV (V12Gy $<10 \mathrm{ml}$ ) was considered just for the single dose of SRS boost.

Doses at $1 \mathrm{ml}$ (D1ml), $2 \%$ (D2), $5 \%$ (D5), $50 \%$ (D50), $95 \%$ (D95), 98 \% (D98), $99 \%$ (D99) and the mean dose to the target volume (Davg) and the volumes to $95 \%$ (V95), $99 \%$ (V99) and $108 \%$ (V108) of the prescribed dose were used for the dosimetric evaluation of PTV boost SRS and SIB.

In order to evaluate homogeneity of the dose distribution and the dose conformity, according to the requirements of ICRU 62 [28], three parameters were analysed:

- Homogeneity Index (HI) is an objective tool to analyse the uniformity of dose distribution in the target volume. Various formulae have been described in literature for its calculation and the more descriptive formula is $\mathrm{HI}=(\mathrm{D} 2-\mathrm{D} 98) / \mathrm{Dp}$, where $\mathrm{D} 2=$ minimum dose to $2 \%$ of the target volume indicating the "maximum dose", D98 = minimum dose to the $98 \%$ of the target volume, indicating the "minimum dose" and $\mathrm{Dp}=$ prescribed dose. This is the most commonly used formula in the literature. The reason for choosing D98 and D2, to represent the minimum and maximum dose, is that the calculation of true minimum or maximum dose is sensitive to the dose-calculation parameters, such as grid size and grid placement, and the high dose gradient is common in Intensity Modulated Radio-Therapy [29]. HI basically indicates the ratio between the maximum and minimum dose in the target volume and the lower value indicates a more homogenous dose distribution within this volume. Smaller HI values indicate more homogeneous dose distributions and the optimal value is to be close to 0 .

Table 1 Organ at risk dose constraints used for treatment plans

\begin{tabular}{lll}
\hline Organ at risk & Dose constraints & \\
\cline { 2 - 3 } & SIB & SRS \\
\hline Brain (without PTV) & $\begin{array}{l}\text { Dmean } \leq 32 \mathrm{GyV} 35 \mathrm{~Gy}<15 \% \\
\text { V40 Gy }<5 \% \text { V45 Gy }<0 \%\end{array}$ & \\
Brainstem & Dmax $<35 G y$ & Excluded \\
Chiasm & Dmax $<35 G y$ & Excluded \\
Optic nerve & Dmax $<35 G y$ & Excluded \\
Lens & Dmax $<5$ Gy & Dmax $<5$ Gy \\
\hline
\end{tabular}


- Conformity Index (CI), defined as the ratio between the total volume enclosed in the references isodose $\left(\mathrm{V}_{\mathrm{RI}}\right)$ and target volume $(\mathrm{TV})\left(\mathrm{CI}=\mathrm{V}_{\mathrm{RI}} / \mathrm{TV}\right)$ $[29,30]$. This index shows the target coverage related to a specific reference isodose (RI) considered as a reasonable limit of acceptability of the treatment plan, in this case the $95 \%$ isodose. If the conformity index is situated between 1 and 2, the treatment is considered to comply with the treatment plan; an index between 2 and 2.5, or 0.9 and 1 , is considered to be a minor violation, and when the index value is less than 0.9 or exceeds 2.5 , the protocol violation is considered to be major, but may nevertheless be considered to be acceptable.

- Conformation Number (CN) is defined as in the next formula: (PTVpi/Vpi) $\times(\mathrm{PTV}$ pi/PTV), where PTVpi is the target volume which is irradiated with prescription isodose (pi) and Vpi is the total volume of tissue which is irradiated with prescription isodose [29]. The values of this index can range between 0 and 1 ; it is usually considered optimal if close to 1 , but values between 0.5 and 0.7 can also be considered satisfactory.

Finally, time of treatment delivery for each planning was calculated.

The statistical analysis was performed using t-Student test for the comparison of the PTV Boost, HI, CI, CN and time of treatment values. The differences were considered statistically significant if $p<0.05$.

\section{Results}

Ten patients were considered for the analysis; for a total number of 13 BM, 52 PTV boost were calculated. The site of disease were cortical lobes, cerebellum and deep structures respectively in 10, 1 and 2 BM. Mean WB-PTV was $1631 \mathrm{cc}$ (range 1275-1899 cc) and mean boost-PTV was $14.32 \mathrm{cc}$ (range 1.43-51 cc). More data are shown in Table 2.

Dose constraints to the OAR evaluated (chiasm, brainstem, right and left lens, right and left optic nerve) were all respected without major violations.

In the SRS cases there was no statistical difference between VMAT and Helical IMRT in terms of respect of OAR, considering that they received the dose due to WBRT and they were basically excluded by the dose contribute of stereotactic boost. In the SIB cases, the OAR were generally better respected in terms of Dmax, particularly for chiasm (30.31 Gy vs 32.36 Gy; $p=0.002$ ) and brainstem (30.95 Gy vs 33.11 Gy; $p=0.013$ ) with Helical IMRT-SIB compared to VMAT-SIB.

The Brain excluded from the PTV volume (Brain-PTV) appear the most critical OAR:
- In the SIB cases there were no major violations in terms of Dmax <32Gy (31.15Gy and 30.96Gy respectively for Helical IMRT and VMAT), V35 < 15Gy (7.65Gy and 4.32Gy respectively), $\mathrm{V} 40<5$ Gy (3.08Gy and 1.37Gy respectively) and V45 < 0 Gy (0.02Gy and 0.11Gy respectively), without statistically significant differences between the techniques;

- In the SRS cases Brain-PTV exceeded the dose constraints (V12 Gy $<10 \mathrm{ml})$ in all VMAT-SRS plans (mean $34.10 \mathrm{ml}$ ) and in 4 Helical IMRT-SRS plans (mean $9.27 \mathrm{ml}$ ), with a statistically significant benefit for Helical IMRT-SRS $(p=0.019)$.

All but six (95\%) of the PTV boosts volumes received at least $95 \%$ of the prescribed dose. The six plans that resulted in inadequate target coverage had been calculated as VMAT-SIB, VMAT-SRS and Helical IMRT-SRS in two cases each respectively. In particular, five of them referred to patients with two BM. Nevertheless, the mean of $V_{\text {PTVboost }} 95 \%$ was over $95 \%$ of prescribed dose for each kind of calculated plans, without statistically significant differences (Table 3).

The analysis of dosimetric parameters for SRS plans showed that HI was 0.07 for both the techniques (very close to the optimal), CI resulted acceptable in VMAT plans (2.1) and optimal in Helical IMRT ones (1.66) without significant differences. $\mathrm{CN}$ appeared better for Helical IMRT than for VMAT (0.66 vs $0.49-p=0,067$ ) (Table 3).

Regarding SIB plans, HI was 0.03 and 0.08 for Helical IMRT and VMAT respectively $(p<0.001)$, no relevant differences emerged for $\mathrm{CI}$ and $\mathrm{CN}$, that resulted within an acceptable range (Table 4).

Time of treatment delivery was significantly longer in Helical IMRT plans (either SRS and SIB, Tables 3 and 4). Helical IMRT-SRS fractions lasted 3 times longer than the VMAT SRS ones (1598 s vs $468 \mathrm{~s}, p<0.001)$. SIB plans were faster to deliver than the SRS ones but still Helical IMRT lasted twice as long (440 s vs $210 \mathrm{~s}, p=0.004)$.

\section{Discussion}

The efficacy and feasibility of a stereotactic boost associated with WBRT has been shown in two randomized clinical trials. In the RTOG 9508 trial [12] 333 patients with 1-3 brain metastases were randomly allocated to either WBRT or SRT-WBRT: WBRT and stereotactic boost treatment improved functional autonomy for all patients and survival for patients with a single metastasis. In the secondary analysis performed after 10 years 252 patients have been re-classified according to the GPA scale and re-analyzed. Survival advantage was 
Table $\mathbf{2}$ Characteristics of cases

\begin{tabular}{|c|c|c|c|c|c|c|c|c|c|c|}
\hline & pt 1 & pt 2 & pt 3 & pt 4 & pt 5 & pt 6 & pt 7 & pt 8 & pt 9 & pt 10 \\
\hline Number of metastases & 1 & 1 & 1 & 2 & 1 & 1 & 1 & 2 & 1 & 2 \\
\hline Site & $\begin{array}{l}\text { Right frontal } \\
\text { lobe }\end{array}$ & $\begin{array}{l}\text { right semioval } \\
\text { center }\end{array}$ & $\begin{array}{l}\text { right parietal } \\
\text { lobe }\end{array}$ & $\begin{array}{l}\text { Right frontal-parietal } \\
\text { lobe and right } \\
\text { occipital lobe }\end{array}$ & $\begin{array}{l}\text { Right occipital } \\
\text { lobe }\end{array}$ & $\begin{array}{l}\text { right lateral } \\
\text { ventricle }\end{array}$ & Cerebellar & $\begin{array}{l}\text { Right occipital lobe } \\
\text { and left parietal lobe }\end{array}$ & $\begin{array}{l}\text { Right temporal } \\
\text { lobe }\end{array}$ & $\begin{array}{l}\text { Right and left } \\
\text { temporal lobe }\end{array}$ \\
\hline Whole brain PTV (ml) & 1796.12 & 1899.90 & 1440.29 & 1573.23 & 1581.73 & 1554.60 & 1769.70 & 1840.10 & 1583.20 & 1275.46 \\
\hline \multirow{2}{*}{$\begin{array}{l}\text { Diameter max. of } \\
\text { metastasis }(\mathrm{mm})\end{array}$} & \multirow[t]{2}{*}{20} & \multirow[t]{2}{*}{13} & \multirow[t]{2}{*}{21} & 20 & \multirow[t]{2}{*}{18} & \multirow[t]{2}{*}{12} & \multirow[t]{2}{*}{30} & 23 & \multirow[t]{2}{*}{13} & 33 \\
\hline & & & & 35 & & & & 8 & & 11 \\
\hline \multirow[t]{2}{*}{ Boost PTV (ml) } & \multirow[t]{2}{*}{10.75} & \multirow[t]{2}{*}{7.25} & \multirow[t]{2}{*}{15.23} & 13.68 & \multirow[t]{2}{*}{11.01} & \multirow[t]{2}{*}{5.77} & \multirow[t]{2}{*}{21.88} & 12.10 & \multirow[t]{2}{*}{5.28} & 25.92 \\
\hline & & & & 51.01 & & & & 1.43 & & 4.79 \\
\hline Number of arcs - SIB & 2 & 3 & 2 & 2 & 2 & 2 & 2 & 4 & 2 & 6 \\
\hline Number of arcs - SRS & 2 & 2 & 2 & 2 & 2 & 2 & 2 & 4 & 2 & 6 \\
\hline
\end{tabular}


Table 3 Dosimetric parameters of SRS plans

\begin{tabular}{|c|c|c|c|c|c|c|c|}
\hline \multirow[b]{2}{*}{ Patient } & \multirow[t]{2}{*}{ Technique } & \multirow{2}{*}{$\begin{array}{l}\text { PTV } \\
\text { D95\% (Gy)/\% }\end{array}$} & Brain-PTV & \multirow[t]{2}{*}{$\mathrm{Cl}$} & \multirow[t]{2}{*}{$\mathrm{HI}$} & \multirow[t]{2}{*}{$\mathrm{CN}$} & \multirow{2}{*}{$\begin{array}{l}\text { Time } \\
\text { (seconds) }\end{array}$} \\
\hline & & & V12Gy (ml) & & & & \\
\hline \multirow[t]{2}{*}{1} & Helical IMRT-SRS & $14.5 / 96.7$ & 9.0 & 2.22 & 0.06 & 0.70 & 1150 \\
\hline & VMAT-SRS & $14.7 / 98.3$ & 0.00 & 2.29 & 0.07 & 0.50 & 386 \\
\hline \multirow[t]{2}{*}{2} & Helical IMRT-SRS & 13.9/92.7 & 6.9 & 1.17 & 0.11 & 0.65 & 1266 \\
\hline & VMAT-SRS & 14.8/98.5 & 36.3 & 3.07 & 0.03 & 0.30 & 419 \\
\hline \multirow[t]{2}{*}{3} & Helical IMRT-SRS & 14.8/98.3 & 10.7 & 1.25 & 0.22 & 0.80 & 1188 \\
\hline & VMAT-SRS & $14.7 / 97.7$ & 58.6 & 3.28 & 0.03 & 0.06 & 375 \\
\hline \multirow[t]{4}{*}{4} & Helical IMRT-SRS A & 14.9/99.3 & 36.2 & 5.54 & 0.04 & 2.53 & 2712 \\
\hline & Helical IMRT-SRS B & $14.6 / 96.7$ & & 1.11 & 0.06 & 0.51 & \\
\hline & VMAT-SRS A & 15.5/103.3 & 13.8 & 1.42 & 0.12 & 1.21 & 543 \\
\hline & VMAT-SRS B & 15.5/103.3 & & 1.12 & 0.13 & 0.90 & \\
\hline \multirow[t]{2}{*}{5} & Helical IMRT-SRS & 14.8/98.7 & 16.4 & 1.48 & 0.04 & 0.46 & 1222 \\
\hline & VMAT-SRS & $15.0 / 100$ & 87.8 & 0.09 & 0.02 & 0.51 & 578 \\
\hline \multirow[t]{2}{*}{6} & Helical IMRT-SRS & 14.8/98.9 & 10.5 & 1.56 & 0.04 & 0.47 & 1051 \\
\hline & VMAT-SRS & $15.0 / 100$ & 59.6 & 6.67 & 0.06 & 0.36 & 549 \\
\hline \multirow[t]{2}{*}{7} & Helical IMRT-SRS & 14.9/99.0 & 1.2 & 1.26 & 0.03 & 0.51 & 1314 \\
\hline & VMAT-SRS & $14.7 / 97.7$ & 26.0 & 1.35 & 0.03 & 0.42 & 425 \\
\hline \multirow[t]{4}{*}{8} & Helical IMRT-SRS A & $13.7 / 91.0$ & 0.50 & 1.00 & 0.13 & 0.27 & 2112 \\
\hline & Helical IMRT-SRS B & $14.7 / 98.1$ & & 1.54 & 0.03 & 0.42 & \\
\hline & VMAT-SRS A & $14.4 / 96.0$ & 16.5 & 1.26 & 0.07 & 0.41 & 571 \\
\hline & VMAT-SRS B & $14.5 / 96.7$ & & 1.70 & 0.04 & 0.44 & \\
\hline \multirow[t]{2}{*}{9} & Helical IMRT -SRS & 14.9/99.5 & 0.54 & 1.57 & 0.01 & 0.37 & 1182 \\
\hline & VMAT-SRS & $14.8 / 98.5$ & 25.1 & 3.00 & 0.01 & 0.33 & 403 \\
\hline \multirow[t]{7}{*}{10} & Helical IMRT-SRS A & $14.5 / 96.3$ & 0.87 & 1.20 & 0.08 & 0.48 & 2784 \\
\hline & Helical IMRT-SRS B & $14.3 / 95.1$ & & 0.69 & 0.09 & 0.38 & \\
\hline & VMAT-SRS A & 14.0/93.3 & 17.4 & 1.10 & 0.13 & 0.34 & 427 \\
\hline & VMAT-SRS B & 14.0/93.3 & & 0.90 & 0.12 & 0.41 & \\
\hline & Helical IMRT-SRS (mean) & 14.55 & 9.27 & 1.66 & 0.07 & 0.66 & 1598.10 \\
\hline & VMAT-SRS(mean) & 14.73 & 34.10 & 2.10 & 0.07 & 0.49 & 467.60 \\
\hline & $\mathrm{p}$ (T student) & ns & 0.019 & ns & ns & ns & 0.000 \\
\hline
\end{tabular}

Legend: Patients number 4, 8 and 10 had two brain metastases, volume and the dosimetric parameters of each lesion (A and $B$ ) are reported in the table

found only in patients with high GPA score (3.5-4) regardless of whether they have 1,2 , or 3 brain metastases. A smaller trial [31] showed that the combination of WBRT and radiosurgery for patients with two to four brain metastases significantly improves control of brain disease, without improvement of survival. Logistically, radiosurgery requires separate localization and treatment procedures that add some inconvenience and cost for patients, providers and caregivers. Single fraction treatments also do not permit the exploitation of the potential radiobiologic benefits of re-assortment and re-oxygenation that may occur with a fractionated radiotherapy course. Hall et al. [22] have argued that fractionated stereotactic radiotherapy may be more efficacious in the treatment of neoplastic disease compared to single fraction radiosurgery.
Additionally, tumour cell repopulation or sub-lethal damage repair may occur if there is a significant break between the radiosurgery and whole brain radiotherapy sessions. Finally, depending on the radiosurgery system used, treatment of more than 3 metastases may involve prohibitively long treatments, requiring multiple sessions or omission of radiosurgery entirely. The introduction of in-room image guidance systems integrated with radiation treatment machines has led to the introduction of non-invasive stereotactic radiosurgery/radiotherapy techniques. One such unit, Helical IMRT combines intensity modulated fan-beam radiotherapy delivery with megavoltage computed tomography (MVCT) imaging for integrated patient positioning and treatment delivery [32, 33]. From a dosimetric point, the ability to incorporate boost contributions 
Table 4 Dosimetric parameters of SIB plans

\begin{tabular}{|c|c|c|c|c|c|c|c|c|c|c|}
\hline \multirow[b]{2}{*}{ Patient } & \multirow[t]{2}{*}{ Technique } & \multirow{2}{*}{$\begin{array}{l}\text { PTV } \\
\text { D95\% (Gy)/\% }\end{array}$} & \multicolumn{4}{|l|}{ Brain-PTV } & \multirow[t]{2}{*}{$\mathrm{Cl}$} & \multirow[t]{2}{*}{$\mathrm{HI}$} & \multirow[t]{2}{*}{$\mathrm{CN}$} & \multirow{2}{*}{$\begin{array}{l}\text { Time } \\
\text { (seconds) }\end{array}$} \\
\hline & & & Dmean (Gy) & V35 (\%) & V40 (\%) & V45 (\%) & & & & \\
\hline \multirow[t]{2}{*}{1} & Helical IMRT-SIB & $44.4 / 98.6$ & 30.6 & 2.4 & 0.85 & 0.00 & 1.61 & 0.02 & 0.31 & 980 \\
\hline & VMAT-SIB & $44.3 / 98.4$ & 31.5 & 3.0 & 1.03 & 0.04 & 1.83 & 0.07 & 0.67 & 277 \\
\hline \multirow[t]{2}{*}{2} & Helical IMRT-SIB & 44.8/99.6 & 31.5 & 12.6 & 5.9 & 0.13 & 4.79 & 0.02 & 0.49 & 247 \\
\hline & VMAT-SIB & $45.1 / 100.3$ & 31.3 & 3.6 & 1.14 & 0.10 & 2.12 & 0.09 & 0.74 & 193 \\
\hline \multirow[t]{2}{*}{3} & Helical IMRT-SIB & 43.9/97.6 & 31.2 & 14.4 & 5.4 & 0.00 & 2.09 & 0.04 & 0.27 & 409 \\
\hline & VMAT-SIB & $42.8 / 95.0$ & 29.8 & 3.4 & 0.83 & 0.01 & 1.11 & 0.16 & 0.68 & 168 \\
\hline \multirow[t]{4}{*}{4} & Helical IMRT-SIB A & $43.5 / 96.6$ & 31.8 & 10.7 & 3.4 & 0.00 & 6.03 & 0.05 & 2.00 & 480 \\
\hline & Helical IMRT-SIB B & $43.4 / 96.5$ & & & & & 0.36 & 0.06 & 0.07 & \\
\hline & VMAT-SIB A & $43.6 / 96.8$ & 32.3 & 16.9 & 5.6 & 0.79 & 8.06 & 0.13 & 3.13 & 195 \\
\hline & VMAT-SIB B & 44.9/99.7 & & & & & 1.68 & 0.12 & 0.71 & \\
\hline \multirow[t]{2}{*}{5} & Helical IMRT-SIB & 44.6/99.1 & 31.4 & 4.3 & 1.55 & 0.00 & 1.83 & 0.02 & 0.46 & 485 \\
\hline & VMAT-SIB & 44.9/99.8 & 31.9 & 3.6 & 1.34 & 0.07 & 3.84 & 0.06 & 0.22 & 237 \\
\hline \multirow[t]{2}{*}{6} & Helical IMRT-SIB & $44.2 / 98.3$ & 31.3 & 4.0 & 0.93 & 0.00 & 1.69 & 0.04 & 0.41 & 401 \\
\hline & VMAT-SIB & 44.9/99.7 & 31.0 & 2.6 & 1.06 & 0.06 & 2.08 & 0.05 & 0.73 & 235 \\
\hline \multirow[t]{2}{*}{7} & Helical IMRT-SIB & $44.2 / 98.2$ & 30.5 & 3.3 & 1.00 & 0.00 & 1.38 & 0.03 & 0.34 & 276 \\
\hline & VMAT-SIB & $43.1 / 95.8$ & 31.3 & 2.8 & 0.90 & 0.00 & 1.11 & 0.05 & 0.00 & 247 \\
\hline \multirow[t]{4}{*}{8} & Helical IMRT-SIB A & $43.8 / 97.3$ & 31.6 & 13.6 & 6.9 & 0.05 & 1.77 & 0.05 & 0.49 & 366 \\
\hline & Helical IMRT-SIB B & $44.4 / 98.6$ & & & & & 3.57 & 0.02 & 0.28 & \\
\hline & VMAT-SIB A & $42.3 / 94.0$ & 29.9 & 3.5 & 0.80 & 0.00 & 1.11 & 0.07 & 0.02 & 149 \\
\hline & VMAT-SIB B & $43.3 / 96.1$ & & & & & 1.58 & 0.04 & 0.01 & \\
\hline \multirow[t]{2}{*}{9} & VMAT-SIB & $43.3 / 99.3$ & 30.3 & 1.5 & 0.50 & 0.00 & 1.19 & 0.05 & 0.15 & 133 \\
\hline & Helical IMRT-SIB & $44.7 / 96.1$ & 30.5 & 3.0 & 1.30 & 0.01 & 2.45 & 0.02 & 0.61 & 372 \\
\hline \multirow[t]{7}{*}{10} & Helical IMRT-SIB A & $44.7 / 99.3$ & 31.0 & 8.2 & 3.6 & 0.00 & 2.12 & 0.02 & 0.54 & 384 \\
\hline & Helical IMRT-SIB B & $44.5 / 98.9$ & & & & & 1.98 & 0.03 & 0.49 & \\
\hline & VMAT-SIB A & $40.9 / 90.9$ & 30.4 & 2.4 & 0.45 & 0.00 & 0.98 & 0.12 & 0.15 & 267 \\
\hline & VMAT-SIB B & $43.3 / 96.3$ & & & & & 1.32 & 0.05 & 0.15 & \\
\hline & Helical IMRT-SIB (mean) & 44.24 & 31.15 & 7.65 & 3.08 & 0.02 & 2.44 & 0.03 & 0.52 & 440.00 \\
\hline & VMAT-SIB(mean) & 43.57 & 30.96 & 4.32 & 1.37 & 0.11 & 2.15 & 0.08 & 0.57 & 210.10 \\
\hline & p T student & ns & ns & ns & ns & ns & ns & 0.000 & ns & 0.004 \\
\hline
\end{tabular}

Legend: Patients number 4, 8 and 10 had two brain metastases, volume and the dosimetric parameters of each lesion (A and B) are reported in the table

with larger field volumes as part of the treatment planning optimization process provides an advantage of the simultaneous boost strategy over sequential whole brain radiotherapy with radiosurgery boost. This advantage occurs as the radiosurgery boost dose is added to the previously delivered whole brain dose without opportunity for optimization of these two components; the need for two different plans potentially results in unintended increased dose to the brain [34].

In the literature there are numerous studies, in which the use of SIB for the treatment of brain metastases was proposed with different techniques. They are all feasibility studies and, sometimes, dose escalation studies with the aim of reaching doses comparable to those of sequential SRS, according to the linear quadratic cube (LQC) model
[20]. The first study was published by Bauman et al. in 2007 [25]: fourteen patients underwent radiotherapy in 10 fraction, $60 \mathrm{~Gy}$ on the lesions and $30 \mathrm{~Gy}$ on whole brain. They choosed this regimen since the dose of 60 Gy in 10 fractions was equated to 18 Gy in single fraction through the LQC model. The comparison to conventional non-coplanar arc fractionated stereotactic radiotherapy plan demonstrated similar target coverage and improved critical tissue sparing even for a challenging anatomy with multiple lesions in the same plane as the optic apparatus. The Authors concluded that the approach with Helical IMRT has potential advantages such as frameless stereotactic localization through daily megavoltage computed tomography, more efficient use of resources and exploitation of radiobiologic advantages of 
fractionation. After that other Authors have proposed different fractionation regimes: Weber [34] judged welltolerated treatments with VMAT in 10 fractions (30 Gy and $40 \mathrm{~Gy}$ on whole brain and on metastatic lesions, respectively); Rodrigues [35] confirmed the data of Bauman [25] with $60 \mathrm{~Gy}$ in $10 \mathrm{fr}$ on metastases with Helical IMRT, after a dose-escalation study from 35 to 60 Gy in $10 \mathrm{fr}$; Zhou [36] proposed WBRT in $20 \mathrm{fr}$ with a total dose of $40 \mathrm{~Gy}$ and during the last week of treatment a simultaneous boost on brain metastases of 20 Gy in $5 \mathrm{fr}$ with IMRT-IGRT.

The sparing of hippocampal areas, during WBRT with volumetric techniques, had already been studied by Gondi [37] in 2010, with the aim of reducing the impact of WBRT on cognitive functions of long-term survivors patients. Recently the use of the SIB with hippocampal sparing has been the object of a greater interest; the aim is to boost the site of disease without interfering with the neurocognitive functions $[38,39]$.

In literature dosimetric comparison trials were not published. With the drawbacks of a limited sample size, our work is therefore the first dosimetric comparative study between techniques (Helical IMRT vs VMAT) and boost delivering modality (SRS vs SIB). Although the number of cases of the present series does not allow to draw clinically sound conclusions, the choice of using a panel of descriptors including conformal index, conformal number and homogeneity index has been made to obtain a synthetic evaluation all the dosimetric issues potentially influential for the cost/benefit ratio of the treatments delivered with the different techniques.

Only one trial has already made a comparison between the different modality of boost administration. Rodrigues et al. [40] in 2013 published a retrospective review of 500 patients treated with SRS alone or SIB-WBRT: no differences in OS were found but SIB was associated with a reduced intracranial failure rate likely due to the WBRT component of the treatment; however, SRS patients did not have WBRT, at variance with the present series. No dosimetric comparison was performed.

\section{Conclusions}

Although limited and preliminary, this series shows that SRS or SIB BM treatments are feasible with both Helical IMRT and VMAT techniques. A notable dosimetric advantage emerged for Helical IMRT plans, in particular to reduce the normal brain irradiated volume (Brain-PTV) in SRS and other critical organs, such as chiasm and brainstem, in SIB. These clinical findings cannot be generalized due to the exiguous number of cases and heterogeneous locations of lesions. No relevant differences were evident for PTV boost coverage, although VMAT plans are more frequently not optimal, in particular for cases with two BM. Mild discrepancies were presented in terms of dosimetric parameters (slightly in favour of Helical IMRT plans, especially for $\mathrm{HI}$ ). This assessment may be due to the intrinsic characteristics of Helical IMRT. In this regard is useful to note that the width of MLC leaves is smaller in Helical IMRT machine than in the Linac used $(0.6 \mathrm{~cm}$ vs $1 \mathrm{~cm})$ and this contributes to increase dose conformation to the target.

A longer treatment delivery time resulted the more important intrinsic limit of Helical IMRT. Too long treatment times should be avoided in order to reduce intrafraction error and patient discomfort.

SIB is a practical way to deliver radiotherapy in BM as an alternative to SRS, since includes WBRT and boost on BM in the same plan. It is therefore characterized by logistic advantages, such as:

- a single simulation, CT-acquisition and elaboration procedure;

- a reduction of waiting times;

- reduced dose delivery time.

All these advantages can be translated in an increased tolerability and treatment compliance for the patient.

In addition, SIB seems to have advantages also from the radiobiological point of view due to the possible reduction of the phenomena of repopulation and redistribution.

In summary, this study suggests that Helical IMRTSIB is able to deliver slightly better plans when compared to VMAT-SIB, but longer treatment times with Helical IMRT-SRS sometimes prohibitive for patients. In these cases SIB plans should be preferred to improve the global outcome of treatment.

\section{Ethical approval and consent to participate}

Not applicable. Our manuscript does not contain sensitive data from human patients. We performed a retrospective analysis on delivered treatment planning and then we recalculated in silico new treatment planning for each boost delivering and radiotherapy techniques, without affection in real administered treatment. All procedures performed in studies were in accordance with the ethical standards of the institutional and/or national research committee and with the 1964 Helsinki declaration and its later amendments or comparable ethical standards.

\section{Consent for publication}

Not applicable.

\section{Availability of data and materials}

Open access dataset on https://zenodo.org/deposit/92796/.

\section{Abbreviations}

BM: brain metastasis; Cl: conformal index; CN: conformal number

Fr: fractions; GPA: graded prognostic analysis; HI: homogeneity index; 
ICRU: International Commission on Radiation Units \& Measurements; KPS: Karnofski performance status; LQ: linear quadratic; LQC: linear quadratic cubic; MLC: multi-leaf collimator; MRI: magnetic resonance; OAR: organ at risk; OS: overall survival; RPA: recursive partitioning analysis; SIB: simultaneous integrated boost; SRS: stereotactic boost; SUR: surgery; TPS: treatment planning system; VMAT: volumetric modulated arc therapy; WBRT: whole brain radiotherapy.

\section{Competing interests}

The authors declare that they have no competing interests.

\section{Authors' contributions}

PB: made substantial contributions to conception and design, acquisition of data, analysis and interpretation of data; been involved in drafting the manuscript, gave final approval of the version to be published and agreed to be accountable for all aspects of the work in ensuring that questions related to the accuracy or integrity of any part of the work are appropriately investigated and resolved. SP: made substantial contributions to acquisition of data, analysis and interpretation of data; she was been involved in drafting the manuscript and agreed to be accountable for all aspects of the work in ensuring that questions related to the accuracy or integrity of any part of the work are appropriately investigated and resolved. LS: made substantial contributions to conception and design and interpretation of data; he was been involved in revising manuscript critically for important intellectual content. RA: made substantial contributions to acquisition of data. MU: made substantial contributions to acquisition of data. FF: she was been involved in drafting the manuscript. GT: made substantial contributions to acquisition and analysis of data. FT: made substantial contributions in analysis and interpretation of data. PG: made substantial contributions in interpretation of data and in drafting the manuscript. SAP: she was been involved in drafting the manuscript. LT: he was been involved in revising manuscript critically for important intellectual content. SMM: made substantial contributions to conception and design; he has been involved in revising manuscript critically for important intellectual content, gave final approval of the version to be published and agreed to be accountable for all aspects of the work in ensuring that questions related to the accuracy or integrity of any part of the work are appropriately investigated and resolved. MB: made substantial contributions to conception and design; she has been involved in revising manuscript critically for important intellectual content, gave final approval of the version to be published and agreed to be accountable for all aspects of the work in ensuring that questions related to the accuracy or integrity of any part of the work are appropriately investigated and resolved. All authors read and approved the final manuscript.

\section{Acknowledgements}

Not applicable.

\section{Funding}

Not applicable.

\section{Author details}

${ }^{1}$ Radiation Oncology Department, University and Spedali Civili Brescia, P.le Spedali Civili 1, Brescia, Italy. ${ }^{2}$ Medical Physics Department, Spedali Civili Brescia, P.le Spedali Civili 1, Brescia, Italy.

\section{Received: 10 January 2016 Accepted: 12 April 2016}

\section{Published online: 19 April 2016}

\section{References}

1. Wn PY, Black PM, Loeffler JS. Metastatic brain cancer. In: De Vita V, Hellma S, Rosenberg SA, editors. Cancer: priciples and practice of oncology. Philadelphia: Lippincott, Williams \& Wilkins; 2001. p. 2655-70.

2. Soffietti R, Rudà $R$, Trevisan E. Brain metastases: current management and new developments. Curr Opin Oncol. 2008. doi:10.1097/CCO.0b013e32831186fe

3. Kushnirsky M, Nguyen V, Katz JS, et al. Time-delayed contrast-enhanced MRI improves detection of brain metastases and apparent treatment volumes. J Neurosurg. 2015;11:1-7.

4. Gaspar $L$, Scott C, Rotman M, et al. Recursive partitioning analysis (RPA) of prognostic factors in three Radiation Therapy Oncology Group (RTOG) brain metastases trials. Int J Radiat Oncol Biol Phys. 1997;37:745-51.
5. Gaspar LE, Scott C, Murray K, Curran W. Validation of the RTOG recursive partitioning analysis (RPA) classification for brain metastases. Int J Radiat Oncol Biol Phys. 2000;47:1001-6.

6. Sperduto PW, Berkey B, Gaspar LE, Mehta M, Curran W. A new prognostic index and comparison to three other indices for patients with brain metastases: an analysis of 1,960 patients in the RTOG database. Int J Radiat Oncol Biol Phys. 2008;70:510-4.

7. Sperduto PW, Shanley R, Luo X, et al. Secondary analysis of RTOG 9508 , a phase 3 randomized trial of whole-brain radiation therapy versus WBRT plus stereotactic radiosurgery in patients with 1-3 brain metastases; poststratified by the graded prognostic assessment (GPA). Int J Radiat Oncol Biol Phys. 2014. doi:10.1016/j.jirobp.2014.07.002.

8. Buglione M, Bandera L, Grisanti S, et al. The impact of tumour histology and recursive partitioning analysis classification on the prognosis of patients treated with whole-brain hypofractionated radiotherapy for brain metastases: analysis of 382 patients. Radiol Med. 2012. doi:10.1007/s11547-011-0738-x.

9. Agnoletti A, Mencarani C, Panciani PP, et al. Surgery in cerebral metastases: are numbers so important? J Cancer Res Ther. 2014. doi:10. 4103/0973-1482.131390.

10. Patchell RA, Tibbs PA, Walsh JW, et al. A randomized trial of surgery in the treatment of single metastases to the brain. N Engl J Med. 1990:322:494-500.

11. Sundström JT, Minn H, Lertola KK, Nordman E. Prognosis of patients treated for intracranial metastases with whole-brain irradiation. Ann Med. 1998;30:296-9.

12. Andrews DW, Scott CB, Sperduto PW, et al. Whole brain radiation therapy with or without stereotactic radiosurgery boost for patients with one to three brain metastases: phase III results of the RTOG 9508 randomised trial. Lancet. 2004:363:1665-72.

13. Weissman DE. Glucocorticoid treatment for brain metastases and epidural spinal cord compression: a review. J Clin Oncol. 1988:6:543-51.

14. Scoccianti S, Ricardi U. Treatment of brain metastases: review of phase III randomized controlled trials. Radiother Oncol. 2012. doi:10.1016/j.radonc. 2011.08.041.

15. Buglione M, Pedretti S, Gipponi S, et al. The treatment of patients with 1-3 brain metastases: is there a place for whole brain radiotherapy alone, yet? A retrospective analysis. Radiol Med. 2015. doi:10.1007/s11547-015-0542-0.

16. Mehta MP, Ahluwalia MS. Whole-brain radiotherapy and stereotactic radiosurgery in brain metastases: what is the evidence? Am Soc Clin Oncol Educ Book. 2015. doi:10.14694/EdBook_AM.2015.35.e99.

17. McDuff SG, Taich ZJ, Lawson JD, et al. Neurocognitive assessment following whole brain radiation therapy and radiosurgery for patients with cerebral metastases. J Neurol Neurosurg Psychiatry. 2013. doi:10.1136/jnnp-2013-305166

18. Kirkpatrick JP, Meyer JJ, Marks LB. The linear-quadratic model is inappropriate to model high dose per fraction effects in radiosurgery. Semin Radiat Oncol. 2008. doi:10.1016/j.semradonc.2008.04.005.

19. Brenner DJ. The linear-quadratic model is an appropriate methodology for determining isoeffective doses at large doses per fraction. Semin Radiat Oncol. 2008. doi:10.1016/j.semradonc.2008.04.004.

20. Joiner MC. Quantifying cell kill and cell survival. In: Joiner MC, van der Kogel A, editors. Basic clinical radiobiology. London: Hodder Arnold; 2009. p. 41-55.

21. Chang EL, Hassenbusch 3rd SJ, Shiu AS, et al. The role of tumor size in the radiosurgical management of patients with ambiguous brain metastases. Neurosurgery. 2003;53:272-80.

22. Hall EJ, Brenner DJ. The radiobiology of radiosurgery: rationale for different treatment regimes for AVMs and malignancies. Int J Radiat Oncol Biol Phys. 1993;25:381-5.

23. Rodrigues $G$, Eppinga W, Lagerwaard F, et al. A pooled analysis of arc-based image-guided simultaneous integrated boost radiation therapy for oligometastatic brain metastases. Radiother Oncol. 2012. doi:10.1016/j.radonc. 2011.05.032

24. Dogan N, King S, Emami B, et al. Assessment of different IMRT boost delivery methods on target coverage and normal-tissue sparing. Int J Radiat Oncol Biol Phys. 2003:57:1480-91.

25. Bauman G, Yartsev S, Fisher B, et al. Simultaneous infield boost with helical tomotherapy for patients with 1 to 3 brain metastases. Am J Clin Oncol. 2007;30:38-44.

26. Lagerwaard FJ, van der Hoorn EA, Verbakel WF, et al. Whole-brain radiotherapy with simultaneous integrated boost to multiple brain metastases using volumetric modulated arc therapy. Int J Radiat Oncol Biol Phys. 2009;75:253-9. 
27. Milano MT, Usuki KY, Walter KA, et al. Stereotactic radiosurgery and hypofractionated stereotactic radiotherapy: normal tissue dose constraints of the central nervous system. Cancer Treat Rev. 2011. doi:10.1016/j.ctrv. 2011.04.004.

28. International Commission on Radiation Units and measurement. ICRU report 62; prescribing, recording and reporting photon beam therapy (supplement to ICRU report 50). Bethesda: ICRU; 1999.

29. Levegrün S, Pöttgen C, Wittig A, et al. Helical tomotherapy for whole-brain irradiation with integrated boost to multiple brain metastases: evaluation of dose distribution characteristics and comparison with alternative techniques. Int J Radiat Oncol Biol Phys. 2013. doi:10.1016/j.jirobp.2013.03.031.

30. Lomax NJ, Scheib SG. Quantifying the degree of conformity in radiosurgery treatment planning. Int J Radiat Oncol Biol Phys. 2003;55:1409-19.

31. Kondziolka D, Patel A, Lunsford LD, Kassam A, Flickinger JC. Stereotactic radiosurgery plus whole brain radiotherapy versus radiotherapy alone for patients with multiple brain metastases. Int J Radiat Oncol Biol Phys. 1999; 45:427-34.

32. Chase D, Ramsey C, Maha S. The impact of inter- and intra-fraction motion in image guided helical tomotherapy. Int J Rad Oncol Biol Phys. 2004;60:619.

33. Mackie TR, Balog J, Ruchala K, et al. Tomotherapy. Semin Radiat Oncol. 1999; 9:108-17.

34. Weber DC, Caparrotti F, Laouiti M, Malek K. Simultaneous in-field boost for patients with 1 to 4 brain metastasis/es treated with volumetric modulated arc therapy: a prospective study on quality-of-life. Radiat Oncol. 2011. doi:10. 1186/1748-717X-6-79.

35. Rodrigues $G$, Yartsev S, Tay KY, et al. A phase II multi-institutional study assessing simultaneous in-field boost helical tomotherapy for 1-3 brain metastases. Radiat Oncol. 2012. doi:10.1186/1748-717X-7-42.

36. Zhou L, Liu J, Xue J, et al. Whole brain radiotherapy plus simultaneous infield boost with image guided intensity-modulated radiotherapy for brain metastases of non-small cell lung cancer. Radiat Oncol. 2014. doi:10.1186/ 1748-717X-9-117

37. Gondi V, Tolakanahalli R, Mehta MP, et al. Hippocampal-sparing whole-brain radiotherapy: a "how-to" technique using helical tomotherapy and linear accelerator-based intensity-modulated radiotherapy. Int J Radiat Oncol Biol Phys. 2010. doi:10.1016/j.jijrobp.2010.01.039.

38. Giaj Levra N, Sicignano G, Fiorentino A, et al. Whole brain radiotherapy with hippocampal avoidance and simultaneous integrated boost for brain metastases: a dosimetric volumetric-modulated arc therapy study. Radiol Med. 2015. doi:10.1007/s11547-015-0563-8.

39. Kim KH, Cho BC, Lee CG, et al. Hippocampus-Sparing Whole-Brain Radiotherapy and Simultaneous Integrated Boost for Multiple Brain Metastases From Lung Adenocarcinoma: Early Response and Dosimetric Evaluation. Technol Cancer Res Treat. 2015. doi:10.1177/1533034614566993.

40. Rodrigues G, Zindler J, Warner A, Bauman G, Senan S, Lagerwaard F. Propensity-score matched pair comparison of whole brain with simultaneous in-field boost radiotherapy and stereotactic radiosurgery. Radiother Oncol. 2013. doi:10.1016/j.radonc.2012.10.014

\section{Submit your next manuscript to BioMed Central and we will help you at every step:}

- We accept pre-submission inquiries

- Our selector tool helps you to find the most relevant journal

- We provide round the clock customer support

- Convenient online submission

- Thorough peer review

- Inclusion in PubMed and all major indexing services

- Maximum visibility for your research

Submit your manuscript at www.biomedcentral.com/submit 